\title{
Adaptive IIR Filters for Active Noise Control
}

\author{
C. Mosquera, J.A. Gómez, F. Pérez, M. Sobreira ${ }^{\dagger}$ and E. Alexandre ${ }^{\dagger}$ \\ Dept. Tecnolox= as das ComunicaciCns, Universidade de Vigo, 36200 Vigo, Spain
}

(Received 9 November 1999; accepted 19 September 2000)

\begin{abstract}
This work is concerned with the performance of adaptive filters with both poles and zeros in active noise control (ANC) applications. This filtered-error type of application makes it necessary to modify classical adaptive algorithms to cancel the noise. A well established theory exists for filtered-error adaptive FIR filters, especially for the FxLMS algorithm. However, when it comes to filtered-error adaptive IIR (Infinite Impulse Response) filters, convergence conditions are not so well understood. IIR filters are specially suited to those scenarios where feedback from the cancelling loudspeaker to the primary source presents high values, and the performance of the cancellation with a fixed filter is not as good as desired. In this work we present the study of the convergence of a family of adaptive IIR algorithms appropriately modified for handling filtered-error situations. Some results based on real measurements are also presented.
\end{abstract}

${ }^{\dagger}$ Member of the International Institute of Acoustics and Vibration (IIAV)

\section{INTRODUCTION}

Conventional methods of suppressing acoustic noise using passive systems such as enclosures, barriers and silencers do not work well at low frequencies. This is because the wavelength is large compared to the system dimensions. In addition, passive methods create problems in airflow systems such as those used for heating, ventilating and air conditioning (HVAC). In an effort to overcome these problems, active noise control (ANC) uses additional secondary sources to cancel the noise created by the primary source, based on the principle of superposition. An antinoise of equal amplitude and opposite phase is created and combined with the primary source, giving as a result the cancellation of both noises. Although first proposed in 1930, ANC did not receive considerable attention until recently, due to the advances in digital signal processing, which permit the execution of sophisticated mathematical functions with enough speed and accuracy in real time. The schematics of an ANC system are shown in Fig. 1. The controller must create the signal $\hat{y}(n)$ to cancel the primary noise at the cancelling loudspeaker by means of destructive interference. The controller is directed by the signal measured at the error microphone; the distance between the error microphone and the cancelling loudspeaker cannot be reduced very much. For short distances the wave cannot be considered as a plane wave, given the presence of higher order modes near the source even below the cut-off frequencies. Those higher order modes can be neglected for distances greater than four times the duct width. We will focus on single channel systems with only a reference microphone and a secondary loudspeaker and adaptive schemes to cancel broadband noise, as typically used in HVAC applications. ${ }^{1}$

The basic principle of the broadband feedforward approach is that the propagation time delay between the upstream microphone and the downstream secondary source offers the possibility of electrically reintroducing the noise downstream to produce cancellation. Figure 2 shows a model of an ANC system where all the analog components have been replaced by their corresponding digital models as a result of sampling. Sampling rates of $1.5-2 \mathrm{kHz}$ are typical in
ANC applications, where the frequency range of interest is below $500 \mathrm{~Hz}$, due to the use of passive devices at higher frequencies. In addition, the sampling rate must be low enough so that the digital circuit processing time per sample $T_{p}$ is less than the sampling period $T$. Causality must be guaranteed for the sampling rate selected, i.e., the acoustic delay from the reference microphone to the secondary speaker must be longer than the electrical delay, formed by the delays in the filters of the $\mathrm{A} / \mathrm{D}, \mathrm{D} / \mathrm{A}$ converters, the delay in the loudspeaker, plus one sampling period $T$. In Fig. $2 H(z)$ denotes the digital version of the path followed by the primary noise from the source to the cancelling or secondary loudspeaker. $S_{1}(z)$ represents the reconstruction filter, the speaker response and the residual transfer function from the loudspeaker to the summing junction. $S_{2}(z)$ models the path common to desired and estimated signals, including the error microphone and the anti-aliasing filter. The response of the reference microphone, together with that of the anti-aliasing filter, are combined in $S_{3}(z)$. The feedback path, microphone and speaker responses, and the filters of the $\mathrm{A} / \mathrm{D}, \mathrm{D} / \mathrm{A}$ converters are combined in $F(z)$. The response of power amplifiers, when present, must also be included at convenient locations. With respect to the classical identification setting, some additional aspects which make this problem more challenging are to be considered:

- Feedback effects. The loudspeaker output radiates upstream to the reference microphone, resulting in a corrupted input signal to the adaptive filter $\hat{H}(z)$. Different solutions can be envisioned for this problem: ${ }^{1}$ directional speakers and microphones, a fixed neutralisation filter, IIR adaptive filters, etc. Due to the broadband character of the problem it is difficult to obtain good directivity over a broad frequency range. We will focus on the use of adaptive IIR filters, since the neutralisation of the effects of $F(z)$ by means of a transfer function $\hat{F}(z)$ identified offline requires continual adjustments of $\hat{F}(z)$ or a robust design, to account for changes in temperature and flow.

- Secondary path effects. In contrast to classical identification schemes, the output of the adjustable filter $\hat{H}(z)$ is not directly subtracted from the desired signal (undesired from the point of view of the application), but it has to go 